\title{
Development of clinical guidelines in a health district: an attempt to find consensus
}

\author{
David Armstrong, Patrick Tatford, John Fry, Pauline Armstrong
}

\begin{abstract}
Objective - To formulate consensus based guidelines for antenatal care in a health district.

Design - Prospective formulation of draft guidelines by a working group of consultant obstetricians and general practitioners with an obstetric interest, canvassing opinions of all GPs in the district by questionnaire, and revision and final circulation of the guidelines.

Setting - One health district.
\end{abstract}

Subjects - All 160 GPs in the district and members of the working party.

Main measures - Questionnaire responses to specific proposals within the draft guidelines for managing anaemia, antepartum haemorrhage, and hypertension.

Results - 136 GPs responded (response rate $85 \%$ ); responders and nonresponders did not differ in age, sex, or presence on obstetric list. Overall they favoured more conservative management than suggested in the guidelines. For example, only $38 \%(44 / 116)$ prescribed iron routinely and $34 \%(38 / 113)$ referred to hospital for haemoglobin concentration of $\leqslant 10 \mathrm{~g} / \mathrm{l} ; 10 \%$ referred women unnecessarily for oedema unassociated with proteinuria; and $20 \%$ managed active bleeding progressing to old brown staining as an urgent admission. The guidelines were revised according to the relative weight of the views obtained.

Conclusion - Establishing guidelines is mainly a political process. Canvassed views influenced guidelines most when internal disagreement existed within the working party.

Implications and action - Regular revising of the guidelines is planned, which, in conjunction with repeating the questionnaire to monitor changing practice, will allow a long term district wide clinical review.

(Quality in Health Care 1992;1:241-244)

\section{Introduction}

A major feature of recent reforms of the NHS is the emphasis given to medical audit to improve quality of care in both primary care and hospital based care. The first step in audit is setting standards against which actual clinical performance can be compared. ${ }^{12}$ Several techniques have been described for generating these clinical guidelines or protocols, ranging from "top down" approaches in which the standards are set by experts, to "bottom up" methods which rely on achieving a consensus among those clincians who will use the standards daily. ${ }^{3}$

The problem with expert based protocols for managing common clinical conditions is the lack of a sense of ownership by those clinicians who have to use them. For this reason they are often reported as having little impact. ${ }^{4}$ This factor is particularly important for clinical guidelines in general practice, which are likely to be based on the views of hospital based consultants: general practitioners (GPs) feel either a lack of commitment or that the advice is not appropriate or relevant to their work.

A major study which tried to enlist the support of GPs for some standardisation of management was undertaken by Irvine $e t a l^{5}$ in the Northern region. Ninety GP trainers met in 10 small groups to set clinical standards for child care, which were then sent out to their practice partners. The use of GPs at all stages of this process ensured relevance for general practice, though each set of guidelines emerged from the deliberations of only relatively few GPs. And, as all participating GPs were drawn from training practices, the best GPs may have been advising the best practices of what constituted good care for the index conditions. However, there is evidence that wider, consensus based guidelines can work in general practice: Grol recently described the successful acceptance by most Dutch GPs of guidelines established through a consultation exercise between experts and a selected panel of 50 GPs. ${ }^{6}$

The research and audit group of the local medical committee in the Bromley Health District, which in the past has actively monitored patterns of general practice work in the district ${ }^{7}$ as well as encouraging improvements in quality of care, decided to draw up clinical guidelines for some common problems: one of these was antenatal care. It was felt from the outset that it was important to involve all GPs in the district with the task, if only to give them a sense of ownership and commitment to the guidelines. The strategy chosen was therefore to incorporate GPs' views on the management of obstetric problems into guidelines which could then be adopted district wide.

\section{Methods}

The process of producing the obstetric guidelines was organised through regular meetings of the research and audit group of 
the local medical committee. Hospital consultants, including an obstetrician, had been invited to join the group as it was intended that any guidelines should be the result of a collaborative effort.

The method involved several stages, as follows.

Production of draft guidelines - A small group of consultant obstetricians and GPs with a special interest in obstetrics was convened to draw up draft guidelines for managing anaemia in pregnancy, antepartum haemorrhage, and hypertension in pregnancy. These guidelines represented the views of the various members of this working party.

Obtaining GPs' comments - The major management points extracted from these draft guidelines were transformed into a questionnaire format. This involved either a simple question or a brief clinical vignette. For example, the guidelines suggested that all women in the health district should be given iron routinely during pregnancy; this recommendation was recast in the question: "Do you usually give routine iron during pregnancy?" The draft guidelines on management of antepartum haemorrhage advised that women with a small haemorrhage several days previously, with only brown staining currently, should be treated at home with bed rest; this recommendation was translated into a question by providing a small vignette of such a case and asking the question: "What would you normally do?" and providing a multiple choice format of responses.

The questionnaire, with a code number to preserve anonymity, was sent to every GP in the health district. A reminder was sent after two weeks to those GPs who had not replied. Basic demographic data on all GPs were obtained from the family practitioner committee so that the non-responders and responders could later be compared.

Revision of the guidelines - GPs' responses were fed back to a joint meeting of the Division of Obstetrics and the GPs from the working party which had drawn up the original draft guidelines, and the guidelines were amended to take account of them.

Distribution of guidelines - The revised guidelines were distributed in specially designed binders to all GPs in the district. They were short and terse so as to offer clear instructions; they were accompanied by an explanatory letter which described the process of their derivation and the sorts of amendments which had resulted in response to the feedback process. The guidelines were also distributed to obstetric consultants and junior hospital doctors to ensure some consistency of policy throughout the district.

\section{Results}

One hundred and thirty six of the 160 GPs in the district returned the questionnaire, a response rate of $85 \%$. Non-responders were no different from responders in terms of age, group, sex, and whether or not they were on the obstetric list.
Table 1 Routine treatment in pregnancy

\begin{tabular}{lcccc}
\hline & Yes & No & Total \\
\cline { 4 - 4 } & No\% & No\% & \\
\hline Do you provide routine iron? & $72(62)$ & $44(38)$ & 116 \\
Do you provide routine folic acid? & $62(54)$ & $53(46)$ & 115 \\
Do you provide routine vitamins? & $42(37)$ & $73(63)$ & 115 \\
\hline
\end{tabular}

The three stages of the process of formulating guidelines are described below for the three obsetric topics: they cover the working party's draft recommendations, the results of the GP survey, and the final revision of the guidelines.

\section{ANAEMIA IN PREGNANCY}

Working party's recommendations - The draft guidelines included recommendations for giving routine iron, folic acid, and vitamins. If the haemoglobin concentration dropped below $10 \mathrm{~g} / \mathrm{l}$ then hospital referral was advised.

Results of survey - Sixty two percent of GPs currently provided iron routinely (table 1 ).

Few GPs identified patients in whom supplements of folic acid should be given. Table 2 shows that most GPs in the district would refer if the haemoglobin concentration fell to $8 \mathrm{~g} / 1$ or less, though only a third would refer at a concentration of $10 \mathrm{~g} / 1$ or less.

Revision of guidelines - In deference to the opinion of those GPs who did not routinely prescribe ion and folic acid, these supplements were advised only when the haemoglobin concentration was less than $12.5 \mathrm{~g} / \mathrm{l}$ and the provision of multivitamin preparations was made optional. It was felt appropriate to provide a full list of patients for whom supplements of folic acid would be suitable. Most GPs in Bromley already gave the recommended preparations - namely, Pregaday, Fefol, and Multivitamin BPC. The original recommendation that patients should be referred if the haemoglobin concentration was $10 \mathrm{~g} / 1$ or less was maintained.

\section{HYPERTENSION IN PREGNANCY}

Working party's recommendations - The draft guidelines advised that when oedema was not associated with proteinuria referral was not necessary. Patients whose blood pressure was greater than $140 / 90 \mathrm{~mm} \mathrm{Hg}$ should be referred to hospital as outpatients, though for blood pressure higher than 150/100 $\mathrm{mm} \mathrm{Hg}$ direct admission should be arranged.

Results of survey - Almost one in 10 GPs said that they would refer a patient with oedema unassociated with proteinuria. About half would refer a pregnant woman with a blood pressure of $140 / 90 \mathrm{~mm} \mathrm{Hg}$ as an outpatient, though the rest would tolerate a higher reading. Similarly, though the draft guidelines suggested a blood pressure of $150 / 100 \mathrm{~mm} \mathrm{Hg}$

Table 2 Referral to hospital with anaemia in pregnancy

\begin{tabular}{ccc}
\hline & Frequency $(\%)$ & Cumulative \% \\
$(n=113)$ & (of all GPs) \\
\hline At what haemoglobin concentration would you & refer to hospital? \\
$\leqslant 11 \mathrm{~g} / 1$ & 5 & 4 \\
$\leqslant 10 \mathrm{~g} / 1$ & 34 & 30 \\
$\leqslant 8 \mathrm{~g} / 1$ & 96 & 85 \\
\hline
\end{tabular}


Table 3 Patterns of management of hypertension in pregnancy among general practitioners. Figures are in numbers of general practitioners

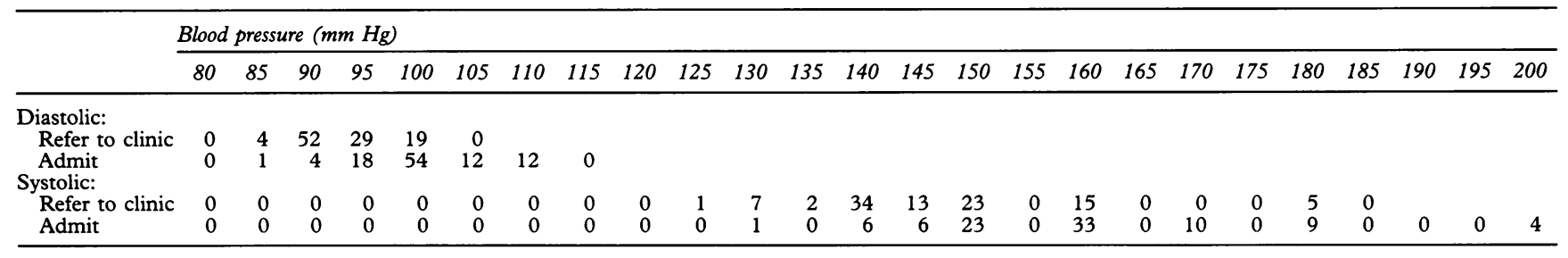

or greater should indicate the need for direct admission, GPs accepted this cut off for diastolic pressure but most would tolerate a systolic pressure of $160 \mathrm{~mm} \mathrm{Hg}$ or greater (table 3).

Revision of guidelines - The final guidelines confirmed that a patient with oedema unassociated with proteinuria need not be referred. Likewise, the original advice on when to refer patients with hypertension was seen to be a safer cut off than current GP practice and therefore was incorporated into the final guidelines.

\section{ANTEPARTUM HAEMORRHAGE}

Working party's recommendations - The guidelines advised that patients with active bleeding which had stopped 48 hours previously and now consisted of old brown staining should be referred to the next clinic but that those with bright red bleeding should be admitted immediately.

Results of survey - Over half of the GPs would manage bleeding which consisted of old brown staining by referring the patients to the next antenatal clinic, $20 \%$ would seek urgent admission, and $12 \%$ would manage by rest and review. Two thirds of GPs would arrange immediate transfer for a woman with bright red bleeding in the 32nd week of gestation.

Revision of guidelines - The final guidelines confirmed the advice provided in the draft.

\section{Discussion}

Creating and maintaining clinical standards can easily be seen as a technical procedure in which the best scientific or medical advice is chosen to inform clinical decision making. In practice, establishing clinical guidelines is primarily a "political" process in that it entails negotiating a common policy between conflicting interests. The procedures reported here illustrate some of this process.

To ensure some degree of ownership of guidelines there was a deliberate attempt to involve doctors throughout the health district. However, there are two interrelated problems in eliciting these many views: firstly, there is still a need to distil a consensus from the broad range of different views that may be expressed and, secondly, the consensus that emerges is in danger of being "mistaken" in terms of scientific criteria.

The use of the term consensus in creating clinical guidelines is, in many ways, a misnomer: it would be unusual for all parties to be in complete agreement on most problems of clinical management. Thus consensus might simply represent majority views, or some formal mechanisms might be instituted to weight different views, though even then different mechanisms can produce different results. ${ }^{8}$ The alternative is to allow the relative value of different views to be based on claims to expertise. This was the main mechanism used in this study, in which a working party consisting of GPs with a special interest in obstetrics and the local consultants drew up the draft recommendations and also, perhaps more importantly, weighed the significance of the views of other GPs in the district. This procedure produced some interesting dynamics. On the one hand, instances occurred when the weight of the GPs' opinion conflicted with the agreed views of the members of the working party, such as when to refer hypertensive patients. In these instances the experts overruled the GPs' views and restated their original clinical advice. GPs' views seemed to influence the working party's decision making when there was already internal disagreement about some policy. For example, there seemed some disagreement among the obstetricians about the value of routine prophylactic iron in pregnancy: the working party looked to the GPs' views for guidance but these were also seriously divided. The solution was a compromise: a recommendation that iron should be given routinely to women with haemoglobin concentrations of less than $12.5 \mathrm{~g} / \mathrm{l}$. This achieved consensus within the working party and, it was thought, represented a middle path for GPs.

Compromises such as this lead to the second problem with consensus based guidelines - namely, their scientific validity. It can be argued that many of the final guidelines were not firmly based on scientific evidence as they emerged from political negotiation rather than discussion of the "evidence." However, scientific evidence does not stand independently of clinician's views but is interpreted and used as part of the everyday repetoire of experts. Such evidence is drawn from particular readings of the formal scientific literature and also from varied clinical experiences. Given that the local working party was drawn from "experts," introducing further expertise, either directly or indirectly through a literature search, would be unlikely to have produced anything other than another political compromise.

Besides illustrating the problems inherent in achieving "valid" and acceptable clinical guidelines, the study did have some important effects. Perhaps the most important was that particular aspects of clinical activity were shifted from the personal conventions of individual clinicians to the exposure of general critical appraisal. Simply being asked to 
explore and agree precise policy helped these aspects to become part of a shared agenda. Secondly, the fact that agreed guidelines did emerge from the negotiations meant that the idea of a common clinical policy has some wider acceptance. Some changes in clinical practice may have resulted from the members of the working party reaching compromises of their own, and GPs throughout the district now have a benchmark against which to compare their own activities.

It is the intention to review and revise these guidelines at regular intervals. In addition, because of the format of this study, it will be possible to repeat the questionnaire survey to establish to what extent the guidelines have succeeded in changing clinical management in the district. It is therefore hoped that a long term procedure of clinical review has been initiated, involving all doctors in the district.
We acknowledge a grant from a primary care initiative by the Nuffield Hospitals Trust, the King's Fund, and the Department of Health, and thank the GPs and obstetricians in the district who contributed so generously to this project.

1 Eddy DM. Practice policies: where do they come from? 7AMA 1990;263:1265-75.

2 Eddy DM. Designing a practice policy; standards, guidelines and options. $7 A M A$ 1990;263:3077-84.

3 Feinstein A, Kosecoff J, Chassin M, Brook RH. Consensus methods: characteristics and guidelines for use $A m .7$ Public Health 1984;74:979-83.

4 Kosecoff J, Kanouse DE, Rogers WH, McCloskey I, Winslow CM, Brook RH. Effect of the National Institutes of Health consensus development program on Institutes of Health consensus development pr
physician practice. $¥ A M A$ 1987;258:2708-13

5 Irvine D, Russell I, Hutchinson A, it al. Educational development and evaluative research in the Northern region. In: Pendleton D, Schofield T, Marinker $M$, eds. In pursuit of quality: approaches to performance review in general practice. London: Royal College of General Practitioners, 1986.

6 Grol R. National standard setting for quality of care in general practice: attitudes of general practitioners and
response to a set of standards. Br $\quad \mathcal{f}$ Gen Pract 1990;40:361-4.

7 Armstrong D, Griffin GA. Patterns of work in the Bromley health district. $尹 R$ Coll Gen Pract 1987;37:264-6.

Scott EA, Black $N$. When does consensus exist in expert panels? F Public Health Med 1991;13:35 9. 